Bull. Mater. Sci., Vol. 3, Number 2, July 1981 pp.

\title{
Use of linear weight factor in real-space renormalization group
}

\author{
D SENGUPTA and $P$ K GHOSH \\ Physics Department, Visva-Bharati University, Santiniketan 731 235, Irdia \\ MS received 15 January 1981
}

\begin{abstract}
An important problem in all real space renormalization grcup calculations is the choice of appropriate weight factor for different ccnfigurations. In this context, the use of linear weight factor has been restricted due to lack of suitable criterion for selecting the parameter $q$. An elegant matching procedure has recently teen developed to fix $q$. First we undertake a systematic investigation of the variation of critical constants with cell size. A comparison is also made with the results obtained using an earlier criterion proposed by Subbarao. Secondly, we extend the applicability of the theory to the realm of dynamic problems by studying the exponent of energylike perturbation in 2D Glauber-Ising model. The value of the exponent obtained for square and triangular lattices are 2.147 and 1.918 respectively which are in good agreement with the general consensus $(\sim 2.0)$.
\end{abstract}

Keywords. Real space renormalization group; linear weight factor; Glauber-Ising model.

\section{Introduction}

Thermodynamic quantities and correlation functions describing a second order phase transition follow power laws and scaling behaviour near the critical point with an universal character. The pioneering studies of Kadanoff (1966) and Wilson (1971) established the renormalisation group ( $R G$ ) analysis which provide physical understanding of such behaviour. The central idea behind the RG technique is a step-by-step decimation of the number of degrees of freedom of the system with incorporation of the effect of decimation in one step into the new Hamiltonian of the next step. For detailed calculations, there are basically two approaches namely the lattice method and the field-theoretic technique. Apart from its inherent conceptual simplicity the lattice method has the advantage of being valid for integer dimensions, including $d=2$ where it can be compared with some exact results. Further, some nonuniversal quantities like the critical temperature $\left(T_{c}\right)$ can easily be calculated in this method.

In the real space renormalization group (RSRG) (Niemeijer and van Leeuwen 1976; van Leeuw. 1978; Wallace and Zia 1978) the reduction in the density of degrees of freedom is accomplished by introducing a new set of spins $\left\{s^{\prime}\right\}$ with larger lattice spacing than the initial set $\{s\}$ and defining a new effective Hamiltonian $H^{\prime}\left(s^{\prime}\right)$. The relation between the initial and the renormalized Hamiltonian is given by 


$$
\exp \left[H^{\prime}\left(s^{\prime}\right)\right]=\sum_{\{s\}} W\left(s^{\prime}, s\right) \exp H(s),
$$

where $W\left(s^{\prime}, s\right)$ is the weight factor having the following properties

(i) $W\left(s^{\prime}, s\right) \geqslant 0$ for all $s^{\prime}, s$,

(ii) $\sum W\left(s^{\prime}, s\right)=1$, which effectively mean that the partition function $Z$ remains $\left\{s^{\prime}\right\} \quad$ invariant under the transformation,

(iii) the symmetries of the Hamiltonian are retained.

The above mentioned restrictions on $W$ still allow an enormous variety in choice for $W$ each leading to a different renormalization transformation. Typically, one chooses a factorisable form for $W$. A widely used choice of the weight factor was introduced by Niemeijer and van Leeuwen (1976) (hereafter NVL) based on the "inajority rule" which associates a new spin $s_{i}$ with suitable group of lattice sites ("cell")

$$
s_{i}^{\prime}=\operatorname{sgn}\left(\Sigma_{i}\right)
$$

where the sum runs over all site-spins in the cell. For triangular lattice where,cellspins have been placed in the centre of three sites, the weight factor has the explicit form

$$
W\left(s^{\prime}, s\right)=\prod_{i} \frac{1}{2}\left[1+s_{i}^{\prime}\left(s_{i}^{1}+s_{i}^{2}+s_{i}^{3}-s_{i}^{1} s_{i}^{2} s_{i}^{3}\right) / 2\right]
$$

with $s_{i}^{a}(\alpha=1,2,3)$ denoting the sites in $i$ th cell. This is an cxample of a nonlinear transformation.

If we now consider the transformation of the interaction parameter (coupling constant) $K$, the renormalized interaction parameter $K^{\prime}$ would be a function of $K$. The nontrivial fixed point that characterizes the critical point requires

$$
K^{\prime}\left(K_{c}\right)=K_{c}
$$

There are a number of approximation schemes for getting reliable estimates of $K^{\prime}(K)$, namely (a) finite lattice, (b) cumulant expansion, and (c) cluster approximation. In this paper, we concentrate on the cumulant expansion scheme.

\section{RSRG with linear weight factor}

In spite of considerable successes, the RSRG method runs into difficulties in specific applications (Brower et al 1977; Kinzel and Fisher 1978). It seems that the nature of errors incurred in the various approximation schemes (Hsu and Gunton 1977) is not clearly understood. This led to suggestions regarding modifications of the original NVL method (Kadanoff 1975). Kadanoff and Houghton (1975) improved the original equal weight scheme of NVL by choosing a nonlinear weight factor optimized by a variational technique. Even then inconsistency remained in fixing the variational parameter (Knops 1977; van Saarloos et al 1978).

The possibility of using linear weight factors was first explored by Subbarao (1975). The explicit form of the linear weight factor is given by 


$$
W_{q}\left(\left\{s^{\prime}\right\},\{s\}\right)=\prod_{a} \frac{1}{2}\left[1+q^{(l)} s_{a}^{\prime} \sum_{i \in c} s_{i}\right]
$$

where $q^{(l)}$ is the adjustable parameter for this transformation with rescaling factor $l$. Every value of $q^{(h)}$ would yield a fixcd point of its own. One is then faced with the problem as to which fixed point to accept. A number of possibilities exists for setting up a proper criterion to fix $q^{(l)}$ (Bell and Wilson 1975).

First, we note that the linear weight factor has the interesting property that its $n$th power, $\left(W_{q}\right)^{n}(n=1,2,3 \ldots \ldots)$, associated with a rescaling factor $l^{n}$, is also linear with the new parameter

$$
q^{\left(l^{n}\right)}=\left[q^{(l)}\right]^{n}
$$

The group structure of block-spin transformation requires that $K_{c}-q^{(l)}$ plots should be identical for cells with rescaling factors $l^{n}$. In actual computations the curves are never coincident. Stella et al (1979) have shown in a variety of cases that the intersections of $K_{c}-q^{(l)}$ curves for $l$ and $l^{2}$ usually provide a reliable estimate not only for $K_{c}^{*}$ but also for the thermal $\left(Y_{T}\right)$ and magnetic $\left(Y_{H}\right)$ exponents. This method would be referred to as intersection method from now on.

On the other hand, Subbarao (1975) showed from a study of the limit of the spinspin correlation function that the existence of a local non-trivial fixed point requires $q^{(l)}$ to be given by

$$
q^{(1)}=3^{-1+(\eta / 4)},
$$

where $\eta$ is the corresponding critical exponent. The magnetic cigenvalue $\lambda_{H}^{(l)}$ is also related to $\eta$ through

$$
\lambda_{H}^{(l)}=3^{1-(\eta / 4)}
$$

Hence for an infinite lattice

$$
\lambda_{H}^{(l)}=1 / q^{(l)} .
$$

Although for a finite lattice this condition is not exactly satisfied, we may use it as a criterion for the choice of $q^{(D)}$.

Equation (9) can also be utilized to establish the relationship between $q^{\left(l_{1}\right)}$ and $q\left(l_{3}\right):$

$$
q^{\left(l_{2}\right)}=\left[q^{\left(l_{1}\right)}\right]^{\left(\log l_{2}\right) /\left(\log l_{1}\right)} .
$$

That (6) follows as a special case of (10) for $l_{2}=\left(l_{1}\right)^{n}$ is obvious. The added advantage of this method is that one need not consider more than one type of cell to arrive at an optimal selection of $q^{(l)}$. 
Table 1. Result of intersection method for planar triangular Ising lattice.

\begin{tabular}{rrrrrrr}
\hline$l^{*}$ & $\ln l^{\prime} / \ln l^{*}$ & $K_{\iota}$ & $q^{(l)}$ & $Y_{T^{(l)}}$ & $Y_{T^{(l)}}$ & $Y_{I I}$ \\
\hline 2 & 1.262 & 0.101 & 0.579 & 0.602 & 0.366 & 1.227 \\
$\sqrt{ }^{7}$ & 1.771 & 0.194 & 0.487 & 0.959 & 0.993 & 1.477 \\
3 & 2.000 & 0.257 & 0.437 & 1.114 & 1.057 & 1.443 \\
$2 \sqrt{3}$ & 2.262 & 0.221 & 0.464 & 1.034 & 1.049 & 1.521 \\
Exact & & 0.275 & & 1.000 & 1.000 & 1.875 \\
\hline
\end{tabular}

$* l=\sqrt{3}$

Table 2. Result of " $1 / \lambda_{H}$ method" for planar triangular Ising lattice.

\begin{tabular}{ccccc}
\hline$l$ & $K_{c}$ & $q$ & $Y_{T}$ & $Y_{H}$ \\
\hline$\sqrt{3}$ & 0.212 & 0.471 & 1.010 & 1.369 \\
2 & 0.218 & 0.424 & 0.699 & 1.238 \\
$\sqrt{7}$ & 0.232 & 0.238 & 1.100 & 1.475 \\
3 & 0.240 & 0.205 & 1.024 & 1.443 \\
$2 \sqrt{3}$ & 0.246 & 0.152 & 1.112 & 1.518 \\
Exact & 0.275 & & 1.000 & 1.875 \\
\hline
\end{tabular}

We now undertake a numerical investigation of the comparative merit of these two techniques.

The approximation used in all the cases is the simple first-order cumulant expansion technique. The calculations are performed for a planar triangular lattice with progressively larger rescaling factors. In the intersection method the comparison is always made with the smallest cell type $(l=\sqrt{ } 3)$. The results for the intersection method and the $1 / \lambda_{H}$ method are summarized in tables 1 and 2 respectively.

In the intersection method, the value of $K_{c}$ shows the closest approach to the exact value for $l^{1}=l^{2}$. Even for a cell with larger rescaling factor $\left(l^{\prime}=2 \sqrt{3}\right)$ the value of $K_{c}$ falls off, thereby establishing the viability of the intersection method though it lacks a firm theoretical basis. The thermal exponent shows reasonable agreement with the exact result for intersections corresponding to cells with large rescaling factors. The values of magnetic exponent are poor in all cases. 
In contrast, the $1 / \lambda_{H}$ method produces a systematic improvement in the value of $K_{c}$ with increase in cell-size. We conclude that computation with a cell of large enough size is capable of yielding a $K_{c}$ close to the exact value. The agreement of $Y_{T}$ with the exact value is in general good. As in the previous method the value of $Y_{H}$ is unreliable.

\section{Critical relaxation in Glauber-Ising model}

A number of rccent theoretical investigations (Achiam 1979; Bolton and Johnson 1976; Rácz and Collins 1976; Suzuki 1977; Yalabik and Gunton 1979) have been able to arrive at a grneral consensus regarding the value of the dynamic exponent for two-dimensional problems within the framework of the Glauber-Ising model (Glauber 1963). The approach to equilibrium is decribed by a time scale $\tau \sim\left|T-T_{c}\right|^{-\Delta}$ where $\Delta$ is the dynamic exponent. The dependence of the relaxation time on the bulk correlation length $\xi$ is characterised by the exponent $z$. The two dynamic exponents arc related by $\Delta=\nu z$ where $\nu$ is the static exponent describing the divergence of $\xi$. The only exact result available is the lower bound of $\Delta: \Delta \geqslant 1.75$ (Abe 1968).

The extended dynamic scaling hypothesis predicts the equality of the index for critical relaxation of energy like perturbation $\left(\Delta_{E}\right)$ and that of magnetic perturbation $\left(\Delta_{M}\right)$. The high-temperaturc expansion gives $\Delta_{M}=2 \cdot 125$ (Rácz and Collins 1976) while Monto-Carlo simulations give $\Delta_{M}=2 \cdot 30$ (Bolton and Johnson 1976) and $\Delta_{E}=2.0$ (Stoll et al 1973). Recently the real-space renormalization group (RSRG) method has been utilized to study these problems. For triangular lattice, Achiam obtained $\Delta_{E}=2.09$ (Achiam 1979a), $\Delta_{M}=2.07$ (Achiam 1978), and for the square lattice $\Delta_{E}=2 \cdot 131$ (Achiam 1979b), $z_{M}=2 \cdot 24$ (Achiam 1980). Kadanoff-Migdal lik; RG transformation leads to a value of $z=1.96$ (Suzuki et al 1979). A recent extensi on of the same method yields $z=1.98$ (Droz and Malaspinas 1980).

In this section we utilize the RSRG analysis to study the decay of energy like perturbation in the first-order cumulant expansion technique with a linear transformation function in contrast to the equal-weight scheme adopted by Achiam.

In the Glauber-Ising model, the relaxation process is constrained by a single spin-flip at a timo, with a transition probability rate $\omega_{i}(\{s\})$. The spin probability distribution $P(\{s\}, t)$ satisfies the empirical master equation

$$
\tau \frac{d P(\{s\}, t)}{d t}=-\sum_{i}\left(1-p_{i}\right) \omega_{i}(s) P(\{s\}, t),
$$

where $p_{i}$ is the spin-flip operator. The master equation can also be written in the general form

$$
\tau \frac{d P(s, t)}{d t}=-\mathscr{P} \phi(s, t)
$$

where $\phi(s, t)=P(s, t) / P(s, t \rightarrow \infty)$, and $\mathscr{L}_{3}$ the Liouville operator, is given by $\mathscr{Z}=\sum_{i} P(s, t \rightarrow \infty) \omega_{i}\left(1-p_{i}\right)$ 
Renormalization of the dynamic problem consists of the static RG transformation followed by a time-rescaling that leaves the master equation of the system invariant. We represent $\phi$ in a perturbation series:

$$
\phi=1+\sum_{a} h_{a}(t) O_{a}(s),
$$

where $O_{a}(s)$ is the time-independent spin operator even under spin-reversal and $h_{a}(t)$ are operators containing their time-dependence. In first order, we retain only $h_{2}$, the nearest-neighbour term. The scaling factor for $\tau$ is given by

$$
\lambda / \omega=l^{2},
$$

where $l$ is the change of length scale by renormalization and $\omega$ and $\lambda$ the scale factors for the time-dependent and time-independent operators respectively.

For the square lattice with four-spin cell $(l=2)$ the condition for fixed point is given by

$$
\begin{aligned}
& 2 f_{1}^{2}=1 \\
& \text { where } f_{1}=\left\langle s_{i}^{a}\right\rangle_{0}=4 q[1+\exp (4 K)] / Z_{0}, \\
& \text { with } Z_{0}=\exp 4 K+6+\exp (-4 K), s_{i}^{a}(i=1,2,3,4),
\end{aligned}
$$

being the site spins of cell $\alpha$. $\lambda$ in equation (14) can be identified with the thermal eigenvalue $\left(\lambda_{T}\right)$ and can be readily evaluated from the expression

$$
\lambda=\left.\frac{d}{d K}\left(2 f_{1}^{2} K\right)\right|_{K=K_{c}} .
$$

Following Achiam, $\phi$ is now expressed in the exponential form

$$
\phi=\exp \left[\frac{1}{2} \sum_{i} s_{i}\left\{h_{2}(t) \sum_{j} s_{j}\right\}\right] .
$$

Retaining only the leading term, we get the renormalized $h_{2}(t)$

$$
h_{2}^{\prime}=2 h_{2} f_{1} Z_{1} / Z_{0},
$$

where $Z_{1}$ is the modified partition function when one spin has all its interactions removed. In the present problem, $Z_{1}$ is given by

$$
Z_{1}=2 q[\exp (2 K)+2+\exp (-2 K)] .
$$

$$
\text { Hence } \omega=\left.\frac{d h_{2}^{\prime}}{d h_{2}}\right|_{K=K_{\varepsilon}}
$$


The intersection of the curves ( $q$ vs $K_{c}$ ) for cells with $l=2$ and $l=4$ gives $q=0.325$ and $K_{c}=0.435$ (Stella et al 1979). This yields

$$
\Delta_{E}=2 \cdot 147
$$

This result is not only above the lower bound of $\Delta$ set by the well-known inequality, but also in close agreement with Achiam's result $\left(\Delta_{E}=2 \cdot 131\right)$, which is obtained using the conventional Niemeijer and van Leeuwen formalism. An exactly similar procedure has been used to study the dynamic problem of planar triangular lattice, giving a value $\Delta_{E}=1.918$. The agreement with Achiam's second-order result $\left(\Delta_{E}=2 \cdot 09\right)$ is gratifying.

\section{Conclusions}

The first-order linear weight scheme has been successfully applied to a series of static RG problems by Stella et al (1979). In this communication we have demonstrated that the same technique is capable of providing reliable results for dynamic problems as well. We have also critically investigated two of the available methods for fixing the parameter $q$ in linear weight factor scheme. The "intersection method" provides reasonable results with limited computational effort whereas " $1 / \lambda_{H}$ method" can give increasingly better result with larger cell size.

\section{References}

Abe R 1968 Prog. Theor. Phys. 39947

Achiam Y 1978 J. Phys. A11 L129

Achiam Y 1979a Phys. Rev. B19 376

Achiam Y 1979b Phys. Lett. A72 35

Achiam Y 1980 J. Phys. A13 1355

Bell T L and Wilson K G 1975 Phys. Rev. B11 3431

Bolton H C and Johnson C H J 1976 Phys. Rev. B13 3025

Brower R C, Kuttner F, Neuenberg M and Subbarao K 1977 Phys. Rev. Lett, 381231

Droz M and Malaspinas A 1980 J. Phys. C13 4365

Glauber R J 1963 J. Math. Phys, 4294

Hsu S and Gunton J D 1977 Phys. Rev. B15 2688

Kadanoff L P 1966 Physics 2263

Kadanoff L P 1975 Phys. Rev. Lett. 341005

Kadanoff L P and Houghton A 1975 Phys. Rev. B11 377

Kinzel W and Fisher K H 1978 J. Phys. C11 2115

Knops H J F 1977 Physica A86 448

Niemeijer Th and van Leeuwen J M J Phase transitions and critical Phenomena Vol. 6(ed.) C Domb and M S Green (London: Academic Press, 1976) 425

Racz Z and Collins M F 1976 Phys. Rev. B13 3025

Stella A L, Dekeyser R, Indekeu J and Toigo F 1979 Phys. Rev. B20 3799

Stoll E, Binder K and Schneider T 1973 Phys. Rev. B8 3266

Subbarao K 1975 Phys. Rev. B11 1165

Suzuki M 1977 Prog. Theor. Phys. 581142

Suzuki M, Sogo K, Ikuo M, Ikeda H, Chikama T and Takano H 1979 Prog. Theor. Phys. 61864

v̀an Leeuwen J M J 1978 Ann. Israel Phys. Soc. 2174

van Saarloos W, van Leeuwen J M J and Pruisken A M M 1978 Physica A92 323

Wallace D J and Zia R K P 1978 Rep. Prog. Phys. 411

Wilson K G 1971 Phys. Rev. B4 3174 and 3184

Yalabik M C and Gunton J D 1979 Prog. Theor. Phys. 621573 